\title{
Automatic selection of localized region-based active contour models using image content analysis applied to brain tumor segmentation.
}

Ilunga-Mbuyamba, Elisee, Avina-Cervantes, J Gabriel, Cepeda-Negrete, J., Ibarra-Manzano, Mario A. y Chalopi, Claire.

Cita:

Ilunga-Mbuyamba, Elisee, Avina-Cervantes, J Gabriel, Cepeda-Negrete, J., Ibarra-Manzano, Mario A. y Chalopi, Claire (2017). Automatic selection of localized region-based active contour models using image content analysis applied to brain tumor segmentation. Computers in Biology and Medicine, 91, 69-79.

Dirección estable: https://www.aacademica.org/jcepedanegrete/13

ARK: https://n2t.net/ark:/13683/pa8v/w38

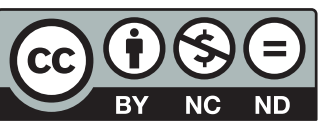




\title{
Automatic selection of localized region-based active contour models using image content analysis applied to brain tumor segmentation
}

\author{
Elisee Ilunga-Mbuyamba ${ }^{\mathrm{a}}$, Juan Gabriel Avina-Cervantes ${ }^{\mathrm{a}, *}$, Jonathan Cepeda-Negrete ${ }^{\mathrm{b}}$, \\ Mario Alberto Ibarra-Manzano ${ }^{\mathrm{a}}$, Claire Chalopin ${ }^{\mathrm{c}}$ \\ ${ }^{a}$ Telematics (CA), Engineering Division (DICIS), Campus Irapuato-Salamanca, University of Guanajuato, Carr. Salamanca-Valle de Santiago km $3.5+1.8 \mathrm{~km}$, \\ Comunidad de Palo Blanco, Salamanca, 36885, Gto., Mexico \\ ${ }^{\mathrm{b}}$ Life Sciences Division (DICIVA), Campus Irapuato-Salamanca, University of Guanajuato, Carr. Irapuato-Silao km 9, ap 311, Ex Hacienda el Copal, Irapuato, 36500, \\ Gto., Mexico \\ ${ }^{\mathrm{c}}$ Innovation Center Computer Assisted Surgery (ICCAS), University of Leipzig, Leipzig, 04103, Germany
}

\section{A R T I C L E I N F O}

\section{Keywords:}

Active contours

Brain tumor segmentation

Automatic feature selection

Image content

2010 MSC:

00-01

99-00

\begin{abstract}
A B S T R A C T
Brain tumor segmentation is a routine process in a clinical setting and provides useful information for diagnosis and treatment planning. Manual segmentation, performed by physicians or radiologists, is a time-consuming task due to the large quantity of medical data generated presently. Hence, automatic segmentation methods are needed, and several approaches have been introduced in recent years including the Localized Region-based Active Contour Model (LRACM). There are many popular LRACM, but each of them presents strong and weak points. In this paper, the automatic selection of LRACM based on image content and its application on brain tumor segmentation is presented. Thereby, a framework to select one of three LRACM, i.e., Local Gaussian Distribution Fitting (LGDF), localized Chan-Vese (C-V) and Localized Active Contour Model with Background Intensity Compensation (LACM-BIC), is proposed. Twelve visual features are extracted to properly select the method that may process a given input image. The system is based on a supervised approach. Applied specifically to Magnetic Resonance Imaging (MRI) images, the experiments showed that the proposed system is able to correctly select the suitable LRACM to handle a specific image. Consequently, the selection framework achieves better accuracy performance than the three LRACM separately.
\end{abstract}

\section{Introduction}

Brain tumor segmentation is commonly used in medicine for quantitative analysis of tumor mass to identify its dynamic growth over time. Many efforts were made in recent years to develop human-free intervention methods that can achieve similar results to those obtained by physicians. Numerous techniques have been used for this purpose, which can be grouped in 2 classes, i.e., supervised [1-6] and unsupervised methods [7-11]. In Refs. [1,2], convolutional neural networks were used for learning and then they were applied to identify abnormal areas. Menze et al. [3] and Cordier et al. [6] employed atlas-based methods to detect brain lesions. By treating the tumor segmentation as a labeling problem [4], the local independent projection-based classification algorithm was used to classify each voxel into different classes. Furthermore, Islam et al. [5] adopted a texture approach for multifractal feature-based brain tumor segmentation. They also suggested the modification of the AdaBoost algorithm to improve tumor tissue classification. Kanas et al. [7] combined the intensity clustering method with Random Walker algorithm to extract neoplastic in MR images. Likewise, the hybrid self-organizing maps with fuzzy $K$-means algorithm was tested by Vishnuvarthanan et al. [8], and they obtained successful tumor identification in their experiments. Since nature-inspired methods became popular in image processing for optimization processes, the active contours driven by the cuckoo search (CS) strategy was introduced in Ref. [9] to lead brain tumor delineation accurately. In addition, the cooperation between Particle Swarm Optimization and $K$-means was suggested in Ref. [10] for segmenting successive slices of 3D MR images. Hamamci et al. [11] have used the tumor probability map that is constructed from cellular automata states to achieve a smooth level set evolution towards brain tumor boundaries delimited in MR images.

\footnotetext{
* Corresponding author.

E-mail addresses: e.ilungambuyamba@ugto.mx (E. Ilunga-Mbuyamba), avina@ugto.mx (J.G. Avina-Cervantes), j.cepeda@ugto.mx (J. Cepeda-Negrete), ibarram@ugto.mx (M.A. Ibarra-Manzano), claire.chalopin@medizin.uni-leipzig.de (C. Chalopin).
} 


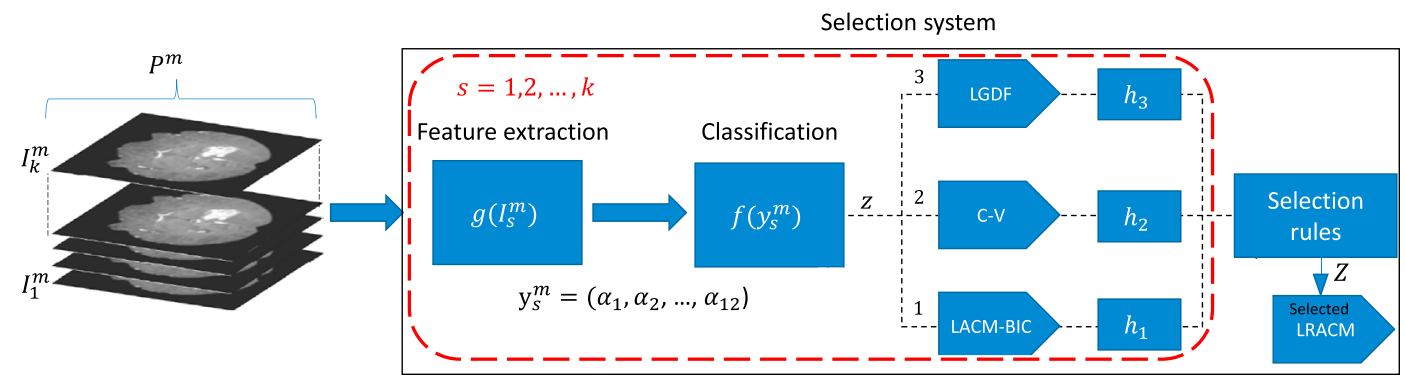

Fig. 1. Flowchart of the LRACM selection for brain tumor segmentation of MRI data. The system selects the LRACM considered as the best for processing the volume $P^{m}$.
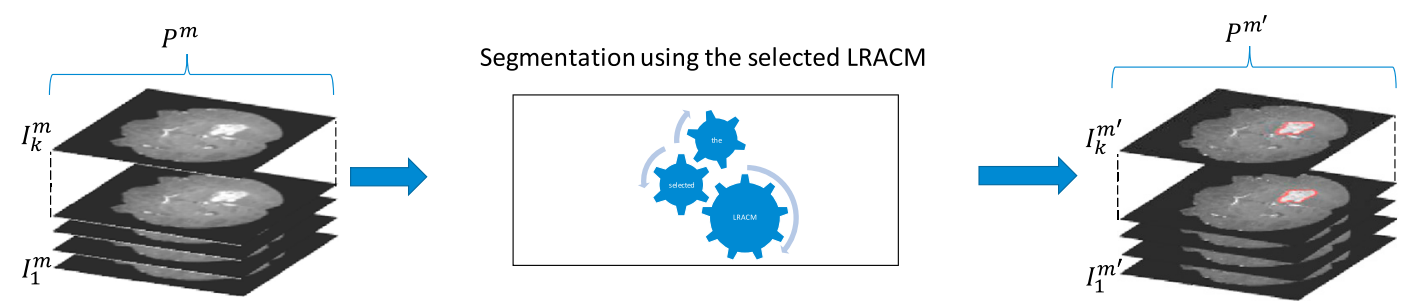

Fig. 2. Concept of image segmentation using a selected ACM. Slices of $P^{m}$ are segmented by using the selected LRACM. $I^{m^{\prime}}$ are the segmented images and $P^{m^{\prime}}$ is the output volume.

Table 1

Statistical features calculated on the $M \times N$ monochromatic image $I(x, y)$ having a histogram $p(i, j)$

\begin{tabular}{|c|c|c|}
\hline Feature & Name & Mathematical description \\
\hline$f_{1}$ & Mean & $\frac{1}{M N} \sum_{x=1}^{M} \sum_{y=1}^{N} I(x, y)$ \\
\hline$f_{2}$ & Harmonic mean & $\frac{M N}{\sum_{x=1}^{M} \sum_{y=1}^{N} 1 / I(x, y)}$ \\
\hline$f_{3}$ & Trimmed mean & $\frac{1}{M_{90 \%} N_{90 \%}} \sum_{x=P_{5}}^{M_{P_{95}}} \sum_{y=P_{5}}^{N_{P_{95}}} I(x, y)$ \\
\hline$f_{4}$ & Maximum value & $\max \{I(x, y)\}$ \\
\hline$f_{5}$ & Median & $\left(\frac{M N+1}{2}\right)^{\text {th }}$ term \\
\hline$f_{6}$ & Standard deviation & $\sqrt{\frac{1}{M N} \sum_{x=1}^{M} \sum_{y=1}^{N}\left(I(x, y)-f_{1}\right)^{2}}$ \\
\hline$f_{7}$ & Skewness & $\sqrt{M N} \frac{\sum_{x=1}^{M} \sum_{y=1}^{N}\left(I(x, y)-f_{1}\right)^{3}}{\left(\sum_{x=1}^{M} \sum_{y=1}^{N}\left(I(x, y)-f_{1}\right)^{2}\right)^{3 / 2}}$ \\
\hline$f_{8}$ & Kurtosis & $M N \frac{\sum_{x=1}^{M} \sum_{y=1}^{N}\left(I(x, y)-f_{1}\right)^{4}}{\left(\sum_{x=1}^{M} \sum_{y=1}^{N}\left(I(x, y)-f_{1}\right)^{2}\right)^{2}}$ \\
\hline$f_{9}$ & Entropy & $-\sum_{i} \sum_{j} p(i, j) \log _{2}(p(i, j))$ \\
\hline$f_{10}$ & Contrast & $\sum_{i} \sum_{j}|i-j|^{2} p(i, j)$ \\
\hline$f_{11}$ & Energy & $\sum_{i} \sum_{j}[p(i, j)]^{2}$ \\
\hline$f_{12}$ & Homogeneity & $\sum_{i} \sum_{j} \frac{p(i, j)}{1+|i-j|}$ \\
\hline
\end{tabular}

Table 2

Data from the global dataset (DB1 + DB2) with details about the patients employed for training and testing the system.

\begin{tabular}{lll}
\hline Class & Patients & \\
\cline { 2 - 3 } & Training & Testing \\
\hline 1 & $4,6,9,11,16,21,20$ & $1,7,17$ \\
2 & $3,8,12,13$ & $5,14,15$ \\
3 & 10,19 & 2,18 \\
\hline
\end{tabular}

An interesting review of medical image analysis for brain tumor studies of MRI data was performed by Bauer et al. [12]. They presented and commented several works concerning registration and segmentation of tumors in MRI. In the same way, a survey of MRI brain tumor segmentation was presented by Gordillo et al. [13]. Manual, semiautomatic, and fully automatic segmentation methods were discussed. Also, many studies based on unsupervised and supervised techniques for brain tumor segmentation were summarized.

Region-based Active Contour Model (RACM) is one of most popular
DB1
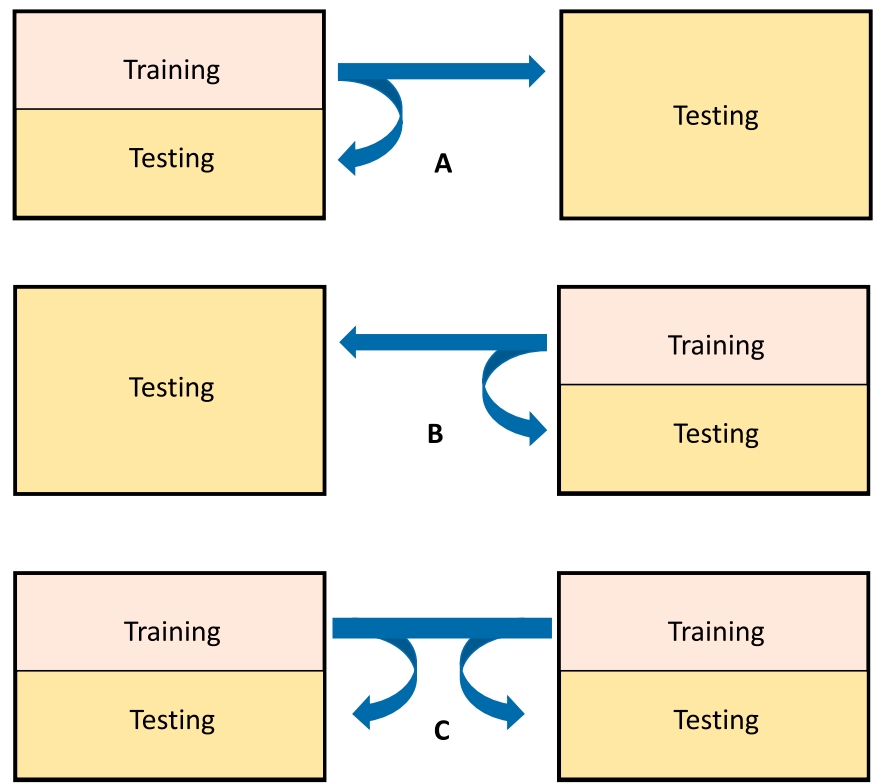

Fig. 3. Three configurations employed for analyzing the system behavior using two databases (DB1 and DB2).

segmentation methods. It uses image content to find the optimum of an energy function that guides the contour evolution in the separation of regions using different statistical information. It has numerous applications in several fields including medicine. For instance, a RACM was used by Saini et al. [14] to detect the atrium and ventricle boundaries in echocardiographic images. They used the Newton-Raphson method to obtain the optimum solution of the evolution equation instead of the gradient descent as in the traditional Chan-Vese model. In Ref. [15], Gupta et al. proposed a RACM initialized by the output of a Gaussian kernel based on a fuzzy C-means clustering method. Their experiments were successfully carried out on ultrasound medical images. Furthermore, a framework combining a machine learning process with the RACM was suggested by Ref. [16] for medical image segmentation. Firstly, selected machine learning algorithms ( $k$-NN and Support Vector Machine) were used to convert images with pixel intensity values to maps 
Table 3

Results presenting the behavior of the selection system by using the RF and $k$-NN classifiers in the three configurations (A, B and C). First, patients are represented by their numbers as properly or badly classified via the representation: Patients correctly selected - Patients wrongly selected. Second, the rates of patients correctly selected per class and configuration are presented.

\begin{tabular}{|c|c|c|c|c|c|}
\hline \multirow[t]{2}{*}{ Config } & \multirow[t]{2}{*}{ Class } & \multicolumn{2}{|c|}{$\begin{array}{l}\text { Patients (correctly — wrongly) } \\
\text { selected }\end{array}$} & \multicolumn{2}{|c|}{$\begin{array}{l}\text { Rate of patients } \\
\text { correctly selected }\end{array}$} \\
\hline & & $\mathrm{RF}$ & $k-\mathrm{NN}$ & $\mathrm{RF}$ & $k$-NN \\
\hline \multirow[t]{4}{*}{ A } & 1 & $\begin{array}{l}1,7-16,17,20, \\
21\end{array}$ & $\begin{array}{l}1,7,16,17 \\
21-20\end{array}$ & $2 / 6$ & $5 / 6$ \\
\hline & 2 & $\begin{array}{l}\times-5,12,13 \\
14,15\end{array}$ & $\begin{array}{l}\times-5,12,13 \\
14,15\end{array}$ & $0 / 5$ & $0 / 5$ \\
\hline & 3 & $2,18,19-\times$ & $2,19-18$ & $3 / 3$ & $2 / 3$ \\
\hline & Average & & & $\begin{array}{l}5 \text { out of } \\
14\end{array}$ & $\begin{array}{l}7 \text { out of } \\
14\end{array}$ \\
\hline \multirow[t]{4}{*}{ B } & 1 & $\begin{array}{l}1,4,6,7,9 \\
11-17\end{array}$ & $\begin{array}{l}4,9,11,17-1,6 \\
7,17\end{array}$ & $6 / 7$ & $4 / 7$ \\
\hline & 2 & $14,15-3,5,8$ & $-5,14,15-3,8$ & $2 /$ & $3 / 5$ \\
\hline & 3 & $\times-2,10,18$ & $2-10,18$ & $0 / 3$ & $1 / 3$ \\
\hline & Average & & & $\begin{array}{l}8 \text { out of } \\
15\end{array}$ & $\begin{array}{l}8 \text { out of } \\
15\end{array}$ \\
\hline \multirow[t]{4}{*}{ C } & 1 & $1,7,17-\times$ & $1,7,17-\times$ & $3 / 3$ & $3 / 3$ \\
\hline & 2 & $14,15-5$ & $14,15-5$ & $2 / 3$ & $2 / 3$ \\
\hline & 3 & $2-18$ & $2-18$ & $1 / 2$ & $1 / 2$ \\
\hline & Average & & & $\begin{array}{l}6 \text { out of } \\
8\end{array}$ & $\begin{array}{l}6 \text { out of } \\
8\end{array}$ \\
\hline
\end{tabular}

of classification probability scores. Secondly, the contour evolution was achieved through the level set method applied to these maps.

Chan and Vese (C-V) [17] proposed a model based on global mean intensity for image approximation. Lankton et al. [18] suggested reformulating this model and the Yezzi model [19], from global to localized methods. The $\mathrm{C}-\mathrm{V}$ and Yezzi energies were referred as Uniform Modeling (UM) and Means Separation (MS) energies. The Lankton method is known as Localizing Region-Based Active Contours and it allows the modeling of any region-based energy of Active Contour Model (ACM) in a localized way. Recently, a specific case of the Lankton Mean Separation model integrating a background intensity compensation stage (LACM-BIC, i.e., Localized Active Contour Model with Background Intensity Compensation) was proposed by Ilunga et al. [20]. This technique showed the improvement of results in image segmentation, especially with large black backgrounds. Moreover, instead of only using mean intensity, Wang et al. [21] proposed the modeling of the local neighborhood by a Gaussian distribution, which is called Local Gaussian Distribution Fitting (LGDF); the contour evolution of their model is based on the mean intensity and on the local region variance.

Selecting the best algorithm among several proposals or its best tuning is a common problem in current studies. Automatic approaches are mostly preferred to manual ones to obtain objective and reliable results. However, this issue is not new and it has already been addressed in many areas. For example, Takemoto and Yokota [22] proposed a framework that selects the optimal algorithm suited to the segmentation of intracellular images. Using supervised techniques, intensity and boundary shapes were extracted as image features. The similarity between feature distribution from user-supervised regions and automatically segmented regions generated by given algorithms was computed to select the optimal algorithm. In the same fashion, the algorithm selection approach for semantic segmentation based on high-level information feedback was presented in Ref. [23] and symbolic segmentation using algorithm selection in Ref. [24]. The automatic selection of the best algorithm is also used for other needs. In Ref. [25] a fuzzy rule-based system was proposed as a selector of color constancy algorithms during the enhancement of dark images. In Ref. [26], Tse et al. used genetic algorithms to automatically select parameters in the design of an optimal complex Morlet wavelet filter, which was applied to bearing fault detection. In the same way, the automatic selection of chemical features for the identification of bioactive molecules in drug discovery was presented by Cano et al. [27].

In the present study, we are concerned with the automatic selection of the localized region-based active contour model applied to the segmentation of brain tumors. The proposed framework considers three LRACM:

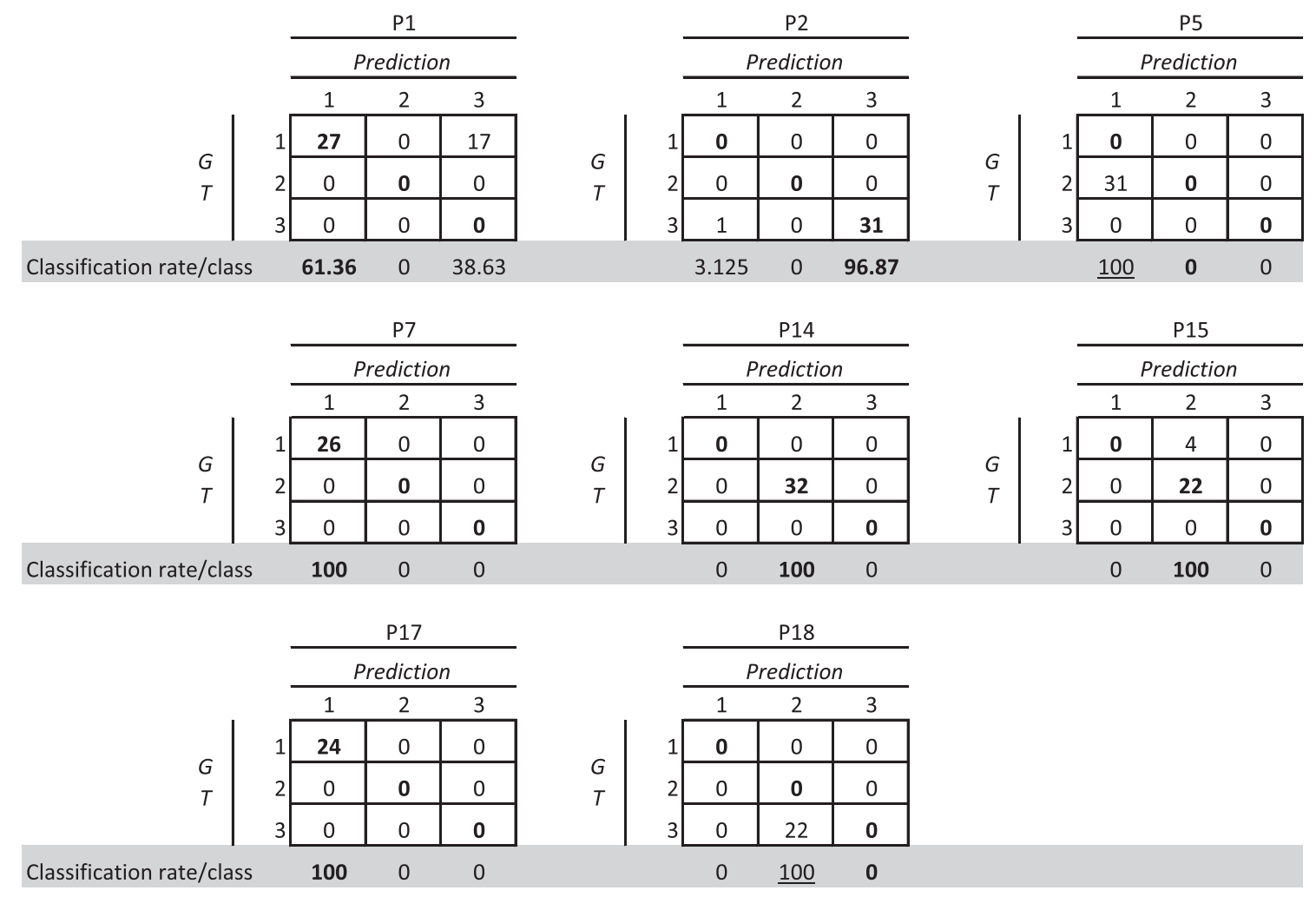

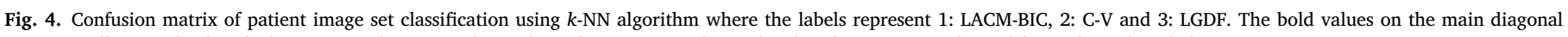
represent all correctly classified instances. The row under each confusion matrix shows the classification rate achieved for each predicted class. 


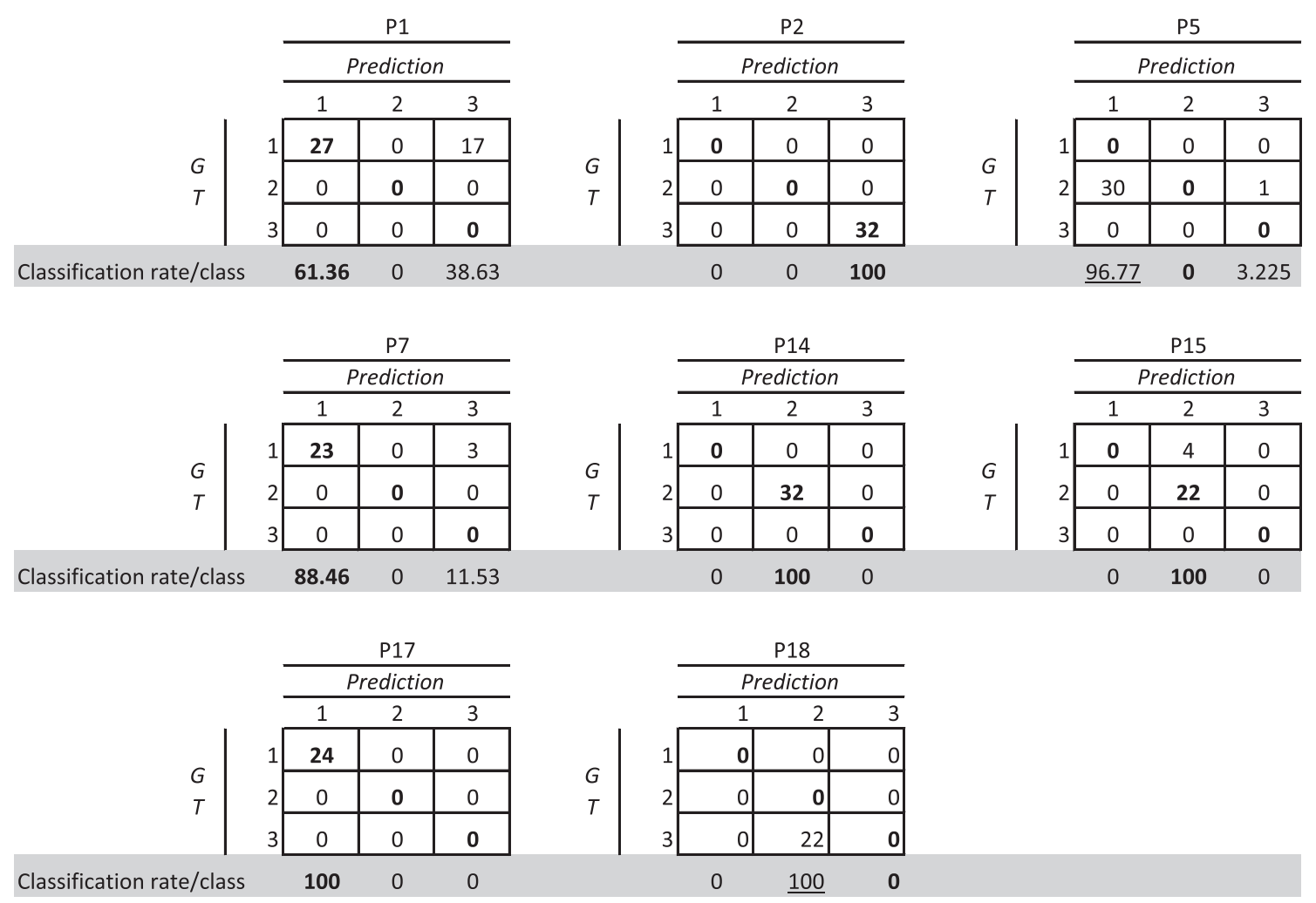

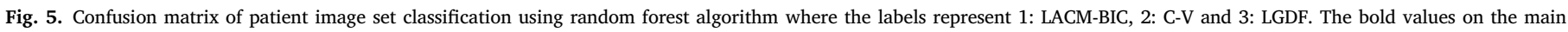
diagonal represent all correctly classified instances. The row under each confusion matrix shows the classification rate achieved for each predicted class.

Table 4

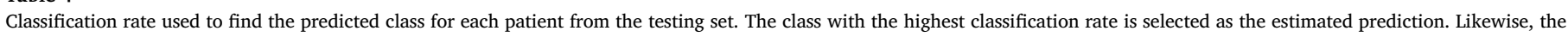

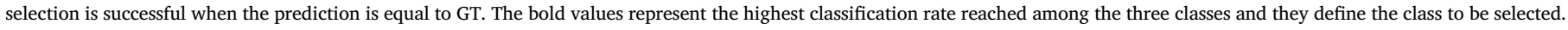

\begin{tabular}{|c|c|c|c|c|c|c|c|c|c|}
\hline \multirow{3}{*}{ Patient } & \multicolumn{3}{|c|}{ Classification rate/class } & \multicolumn{3}{|c|}{ Classification rate/class } & & & \multirow{3}{*}{$\overline{\mathrm{GT}}$} \\
\hline & \multicolumn{3}{|l|}{$\mathrm{RF}$} & \multicolumn{3}{|l|}{$k$-NN } & \multicolumn{2}{|c|}{ Predictions } & \\
\hline & 1 & 2 & 3 & 1 & 2 & 3 & $\mathrm{RF}$ & $k$-NN & \\
\hline 1 & 61.36 & 0.000 & 38.63 & 61.36 & 0.000 & 38.63 & 1 & 1 & 1 \\
\hline 2 & 3.125 & 0.000 & 96.87 & 0.000 & 0.000 & 100 & 3 & 3 & 3 \\
\hline 5 & 100.0 & 0.000 & 0.000 & 96.77 & 0.000 & 3.225 & 1 & 1 & 2 \\
\hline 7 & 100.0 & 0.000 & 0.000 & 88.46 & 0.000 & 11.53 & 1 & 1 & 1 \\
\hline 14 & 0.000 & 100.0 & 0.000 & 0.000 & 100.0 & 0.000 & 2 & 2 & 2 \\
\hline 15 & 0.000 & 100.0 & 0.000 & 0.000 & 100.0 & 0.000 & 2 & 2 & 2 \\
\hline 17 & 100 & 0.000 & 0.000 & 100.0 & 0.000 & 0.000 & 1 & 1 & 1 \\
\hline 18 & 0.000 & 100.0 & 0.000 & 0.000 & 100.0 & 0.000 & 2 & 2 & 3 \\
\hline
\end{tabular}

the LGDF [21], the C-V in the Lanktom formulation [18] and LACM-BIC [20] models. Used in Ref. [20], they achieved best results for complex images of specific patients. In consequence, the automatic selection of one of these techniques based on the image content is suggested. The aim of this work is (1) to show that object segmentation using ACM is image content dependent and (2) to execute the segmentation task using the selected model that may achieve best results for a given patient's images. The system architecture has two main stages. The first consists in a learning process, where a part of the data is used to train the system in the selection task of the best LRACM. Twelve image features were computed for this purpose: mean, harmonic mean, trimmed mean, maximum value, median, standard deviation, skewness, kurtosis, entropy, contrast, energy, and homogeneity. The second comprises the assessment, where the remainder data were tested to evaluate the ability of the proposed system to correctly select the expected active contour model. Two classifiers were employed to carry out these experiments, $k$-NN and random forest. In fact, the goal is not to focus on the choice of the best classifier, but it is rather to show that the success of the most representative LRACM proposed in the literature is related to the image content. Hence, an adequate choice to handle input images ensures that the system will reach the highest segmentation accuracy for all instances. It is important to note that this work is different from Ref. [20]. The former compared the ACM performances while this study proposes an alternative ACM selection system.

In the previous work, it was observed that the ACM behavior can change for specific patients and this hypothesis is analyzed and discussed in this paper. Accordingly, the design of the system for the ACM automatic selection is described in Section 2. Experiment results are provided and analyzed in Section 3. The results are discussed in Section 4, and finally, Section 5 presents the conclusions of this work.

\section{Method and materials}

\subsection{Automatic selection of LRACM based on image content}

\subsubsection{System description}

The proposed system is based on the fact that the outcomes reached 
Table 5

Accuracy comparison of each patient from the testing set with different methods by using Dice and Hausdorff distance metrics. Bold values represent the best results given by the metrics among the tested methods for each patient.

\begin{tabular}{lllllll}
\hline \multirow{2}{*}{ Patient } & Metric & $\begin{array}{l}\text { LGDF } \\
{[21]}\end{array}$ & $\begin{array}{l}\text { C-V } \\
{[18]}\end{array}$ & $\begin{array}{l}\text { LACM-BIC } \\
{[20]}\end{array}$ & $\begin{array}{l}\text { Selection } \\
(\mathrm{RF})\end{array}$ & $\begin{array}{l}\text { Selection } \\
(k-\mathrm{NN})\end{array}$ \\
\hline \multirow{2}{*}{1} & $\mathrm{D}$ & 0.8159 & 0.7833 & $\mathbf{0 . 9 6 1 8}$ & $\mathbf{0 . 9 6 1 8}$ & $\mathbf{0 . 9 6 1 8}$ \\
& $\mathrm{d}_{H}$ & 3.3200 & 5.2736 & $\mathbf{2 . 3 6 7 8}$ & $\mathbf{2 . 3 6 7 8}$ & $\mathbf{2 . 3 6 7 8}$ \\
2 & $\mathrm{D}$ & $\mathbf{0 . 9 4 0 1}$ & 0.9246 & 0.9077 & $\mathbf{0 . 9 4 0 1}$ & $\mathbf{0 . 9 4 0 1}$ \\
& $\mathrm{d}_{H}$ & $\mathbf{1 . 8 2 0 2}$ & 1.8403 & 1.8571 & $\mathbf{1 . 8 2 0 2}$ & $\mathbf{1 . 8 2 0 2}$ \\
5 & $\mathrm{D}$ & 0.6987 & $\mathbf{0 . 9 7 3 8}$ & 0.9721 & 0.9721 & 0.9721 \\
& $\mathrm{~d}_{H}$ & 3.2288 & $\mathbf{1 . 9 3 4 8}$ & 2.0021 & 2.0021 & 2.0021 \\
7 & $\mathrm{D}$ & 0.7781 & 0.8203 & $\mathbf{0 . 8 6 1 9}$ & $\mathbf{0 . 8 6 1 9}$ & $\mathbf{0 . 8 6 1 9}$ \\
& $\mathrm{d}_{H}$ & 2.8781 & 2.6834 & $\mathbf{2 . 4 0 1 2}$ & 2.6834 & $\mathbf{2 . 4 0 1 2}$ \\
14 & $\mathrm{D}$ & 0.8276 & $\mathbf{0 . 8 8 0 8}$ & 0.8704 & $\mathbf{0 . 8 8 0 8}$ & $\mathbf{0 . 8 8 0 8}$ \\
& $\mathrm{d}_{H}$ & 6.9270 & $\mathbf{6 . 2 0 0 6}$ & 6.2592 & $\mathbf{6 . 2 0 0 6}$ & $\mathbf{6 . 2 0 0 6}$ \\
15 & $\mathrm{D}$ & 0.8381 & $\mathbf{0 . 9 2 6 1}$ & 0.9163 & $\mathbf{0 . 9 2 6 1}$ & $\mathbf{0 . 9 2 6 1}$ \\
& $\mathrm{d}_{H}$ & 4.3222 & $\mathbf{3 . 1 4 6 9}$ & 3.2573 & $\mathbf{3 . 1 4 6 9}$ & $\mathbf{3 . 1 4 6 9}$ \\
17 & $\mathrm{D}$ & 0.9598 & 0.9600 & $\mathbf{0 . 9 6 1 4}$ & $\mathbf{0 . 9 6 1 4}$ & $\mathbf{0 . 9 6 1 4}$ \\
& $\mathrm{d}_{H}$ & 3.6464 & 3.7614 & $\mathbf{3 . 7 0 3 6}$ & $\mathbf{3 . 7 0 3 6}$ & $\mathbf{3 . 7 0 3 6}$ \\
18 & $\mathrm{D}$ & $\mathbf{0 . 9 6 9 5}$ & 0.9516 & 0.9465 & 0.9516 & 0.9516 \\
& $\mathrm{~d}_{H}$ & $\mathbf{2 . 2 7 1 9}$ & 2.3774 & 2.4982 & 2.3774 & 2.3774 \\
\hline
\end{tabular}

Table 6

Average and standard deviation of similarity measures: the Dice index and Hausdorff distance. Bold values represent the best results among the tested methods.

\begin{tabular}{lll}
\hline \multicolumn{2}{l}{ Average and standard deviation of similarity measures } \\
\hline Methods & Dice coefficient & Hausdorff distance \\
\hline LBF [38] & $0.6987 \pm 0.1789$ & $4.4781 \pm 1.9980$ \\
LGDF [21] & $0.8535 \pm 0.0958$ & $3.5518 \pm 1.5695$ \\
C-V [18] & $0.9026 \pm 0.0690$ & $3.4023 \pm 1.5896$ \\
LMS [18] & $0.9172 \pm 0.0386$ & $3.1832 \pm 1.3517$ \\
LACM-BIC [20] & $0.9248 \pm 0.0426$ & $3.0433 \pm 1.4383$ \\
Selection system & $\mathbf{0 . 9 3 2 0} \pm \mathbf{0 . 0 4 0 3}$ & $\mathbf{3 . 0 0 2 5} \pm \mathbf{1 . 4 2 9 0}$ \\
\hline
\end{tabular}

by different LRACM, under the same conditions, are not thesss same. For instance, let $\Omega$ be the global dataset s.t. $\Omega=A_{1} \cup A_{2} \ldots \cup A_{i}$ where $A_{i}$ represents the image subsets of $\Omega$ and $i$ the subset index. It is observed that one method can be better than the others for the given subset $A_{1}$ and another one could be the best for subset $A_{2}$ and so on. Likewise, the segmentation results achieved in Ref. [20] showed that the LACM-BIC outperforms in general comparative methods. However, for the images of the considered patients, the Localized C-V [18] and LGDF [21] methods reached the best results in three and two patient cases, respectively. Therefore, the automatic selection task of an active contour model for a given input image set can improve the segmentation outcomes. Hence, a system that has to perform this selection based on supervised learning techniques is proposed. It consists of two main stages, training and testing. In the same fashion, the dataset used is split into two parts, namely, training and testing sets. Sixty percent of dataset images were used for training and the remainder forty percent for testing the designed system. Data are grouped into three classes or subsets $A_{i}$ $(\forall i=1,2,3)$ based on the ACM segmentation results achieved in Ref. [20]. Let $I$ denote an input image, the selection of the algorithm with the best expectation performance is achieved by extracting the first $n$ image features $\left\{\alpha_{j}\right\}_{j=1}^{n}$ from $I$. Then, a classifier is employed to analyze the feature vector $\mathbf{y} \in \Re^{n}$ in order to predict the class to which each $I$ belongs. Any classifier can be integrated into this framework, but currently, the experiment will be carried out using the $k$-NN and random forest (RF) classifiers. The system receives an input image $I$, then it extracts the vector $\mathbf{y}$ that includes twelve image features $(\mathbf{y}=$ $\left.\left(\alpha_{1}, \ldots, \alpha_{n}\right), n=12\right)$ to describe the image content. Lastly, the system has an output $Z \in\{1,2,3\}$ that corresponds to the label of the selected LRACM (1: LACM-BIC, 2: C-V and 3: LGDF).

Moreover, in the design of the system, it is assumed that 2D images of a $3 \mathrm{D}$ volume acquired from a specific patient (i.e., under the same conditions, with the same scanner, imaging protocol, and acquisition parameters) share the same features. Consequently, they can be grouped in the same subset $A_{i}$ for which one LRACM should be automatically selected. In supervised learning, $A_{i}$ is known as the actual class or ground truth (GT). Even if each 2D slice image is classified separately in a predicted class $C_{i}$ via a label $z \in\{1,2,3\}$, the selection rule of the LRACM should be carried out by the system in function of the $3 \mathrm{D}$ volume (image patient dataset). Let $P^{m}$ be the image set of the $m$-th patient and $h_{i}^{m}$ the number of samples (slices) in a predicted class $C_{i}^{m}$ subject to

$P^{m}=\left\{I_{1}^{m}, \ldots, I_{k}^{m}\right\}$

$C_{i}^{m}=\left\{I_{k}^{m} \mid g: I_{k}^{m} \rightarrow \mathbf{y}_{k}^{m} \quad\right.$ and $\left.\quad f: \mathbf{y}_{k}^{m} \rightarrow z_{\mid z=i}\right\}$

$h_{i}^{m}=\# C_{i}^{m}$

where, $k$ is the number of $2 \mathrm{D}$ images $I^{m}$ in the volume $P^{m}, \# C_{i}^{m}$ the cardinality of $C_{i}^{m}, g$ the function that extracts feature vectors $\mathbf{y}_{k}^{m}=$ $\left\{\mathbf{y}_{1}^{m}, \ldots, \mathbf{y}_{k}^{m} \mid g: I_{k}^{m} \rightarrow \mathbf{y}_{k}^{m}\right\}$ and $f$ the decision function that attaches to $\mathbf{y}_{k}^{m}$ a class $z$. Two notations of labels are used, $z$ is the label of a slice $I_{k}^{m}$ (or partial label), while $Z$ is the final label assigned to the volume $P^{m}$. Fig. 1 describes the selection process of the LRACM for brain tumor segmentation of MRI data. Firstly, based on selection rules, the proposed system selects the LRACM considered as the best for handling the volume $P^{m}$. Secondly, slices of $P^{m}$ are segmented by using the selected LRACM as is depicted in Fig. 2.

The class membership of $P^{m}$ will be performed by using a majority vote among images $I_{k}^{m},(m=1, \ldots, k)$, i.e., by looking for the largest $h_{i}^{m}$. This last part can be found by computing the index of the most populous predicted class as

$q=\underset{z \in\{1,3\}\}}{\arg \max }\left\{h_{z}^{m}\right\}$

Index $q$ can be computed by using the prediction classification rate as

$q=\underset{z \in\{1,2,3\}}{\arg \max }\left\{h_{z}^{m} / \sum_{i=1}^{3} h_{i}^{m}\right\}$

For example, the label $Z=1$ will be attached to $P^{m}$ if $q=1$, which means when

$h_{1}^{m}>h_{2}^{m} \quad \cap \quad h_{1}^{m}>h_{3}^{m}$

or by using the prediction classification rate as

$h_{1}^{m} / \sum_{i=1}^{3} h_{i}^{m}>h_{2}^{m} / \sum_{i=1}^{3} h_{i}^{m} \cap h_{1}^{m} / \sum_{i=1}^{3} h_{i}^{m}>h_{3}^{m} / \sum_{i=1}^{3} h_{i}^{m}$

These last two rules (Eqs. (6) and (7)) are equivalent and they will lead to the same result. However, the former equation (based on Eq. (4)) is simpler and faster than the second one in implementation, while the latter (based on Eq. (5)) is preferred when the classification rate evaluation is needed. In special cases, when no class receives a majority of votes $h_{i}^{m}$, the selection rule will assign to $P^{m}$ a class membership based on prior probability. This last part is related to the class size. Moreover, by referring to Table 2, it is clear that the size of class 1 is the biggest and the size of class 2 is bigger than that of class 3. To illustrate this, if $h_{1}^{m}=$ $h_{2}^{m}>h_{3}^{m}$ or $h_{1}^{m}=h_{3}^{m}>h_{2}^{m}$ or $h_{1}^{m}=h_{2}^{m}=h_{3}^{m}$, the selection rule will assign to $P^{m}$ a class membership $Z=1$. Besides, if $h_{2}^{m}=h_{3}^{m}>h_{1}^{m}$, a label $Z=2$ will be then attached to $P^{m}$.

The selection rules of the proposed system can be implemented by using the following algorithm:

Algorithm 1. Algorithm of the proposed LRACM selection system where selection rules are described. 
Data: Parameters such as total patient number $\left(p_{\max }\right)$, current patient volume $\left(P^{m}, m=1, \ldots, p_{\max }\right)$, total slice number $(k)$, current slice $\left(I_{s}^{m}, s=1, \ldots, k\right)$, slice classification label $(z)$ and the class index $(i)$

Result: the patient classification label $(Z)$

Initialize $m=1, s=1, C_{i}^{m}=0, h_{i}^{m}=0$

while $\left(m \leq p_{\max }\right)$ do

Read volume $P^{m}$

Extract $k$

while $(s \leq k)$ do

Extract feature vector $\mathbf{y}_{s}^{m}$

Classify $\mathbf{y}_{s}^{m}$ by using the decision function $f$, s.t. $f: \mathbf{y}_{k}^{s} \rightarrow z$ via a classifier

Update $C_{i}^{m}$ by using Eq. (2) with $i=z$

end

for $i=1,2, \ldots, 3$ do

| Update $h_{i}^{m}=\# C_{i}^{m}$, see Eq. (3)

end

Find $q$, the index of the largest $h_{z}^{m}$, by using Eq. (4) or Eq. (5)

Find the subset $w$ that satisfy $h_{q}^{m}=h_{z}^{m}$, s.t. $z \in w \subseteq\{1,2,3\}$

if $z=1 \in w$ then

I $Z=1$

else if $z=2 \in w$ then

I $Z=2$

else

l $Z=3$

end

$m=m+1$

end

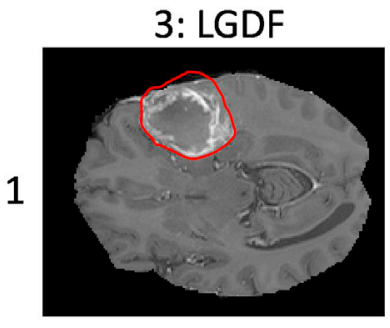

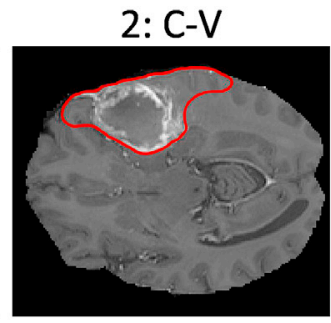

3: LACM-BIC
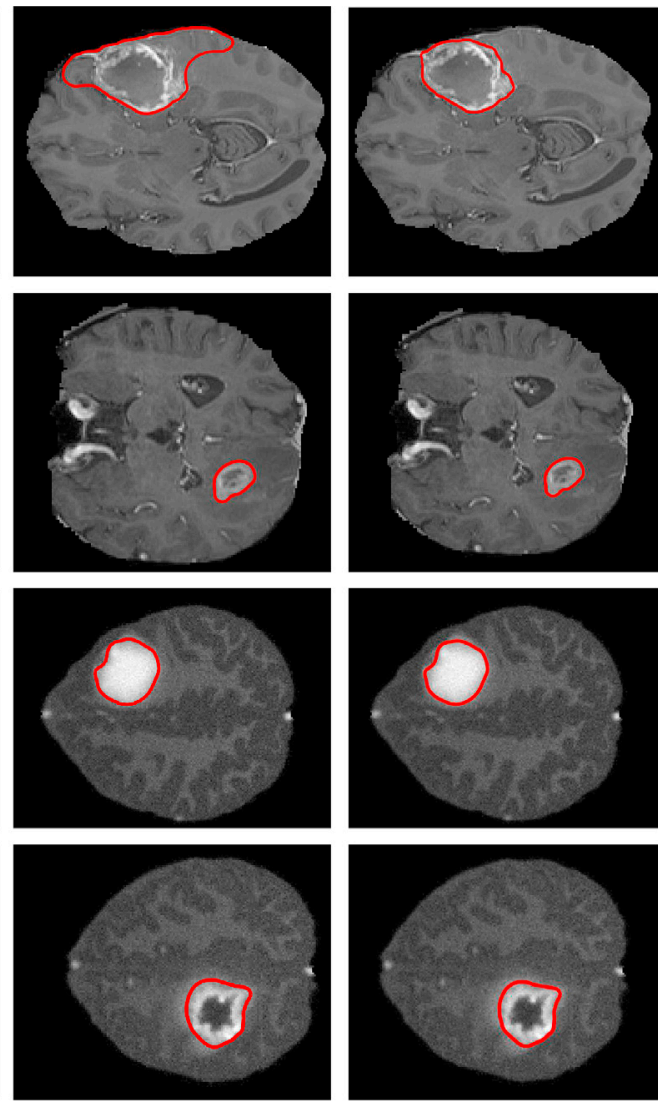
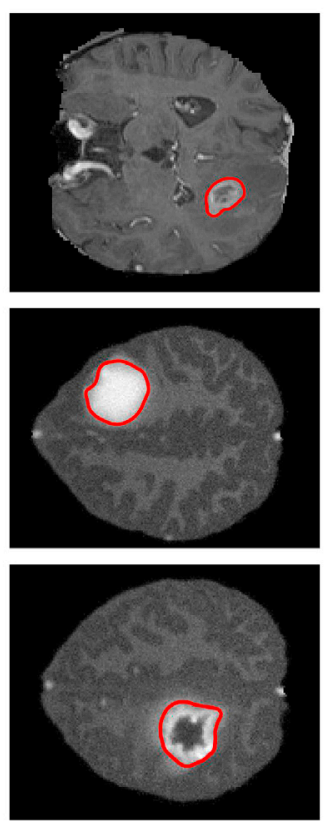
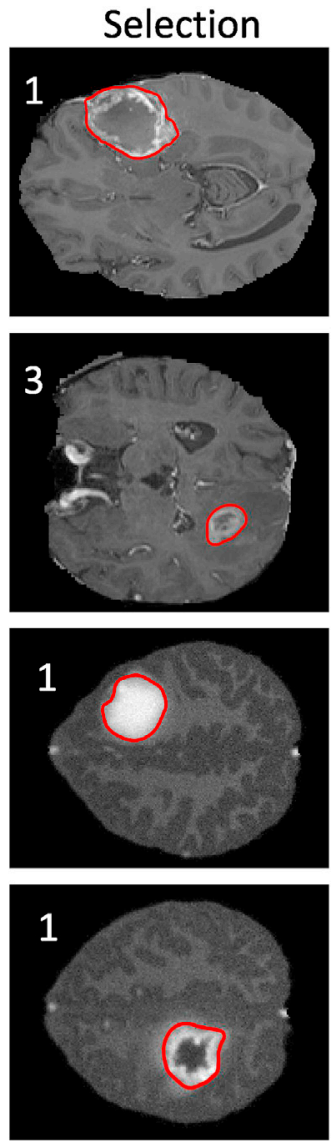

Fig. 6. Segmentation results of MRI data: patients 1, 2, 5 and 7. From left to right, the LGDF, localized C-V, LACM-BIC and the proposed algorithm. 
3: LGDF

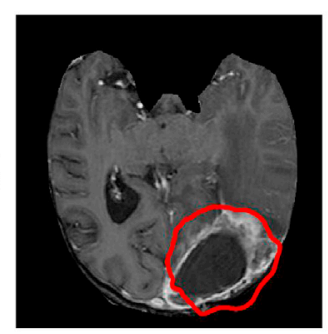

14
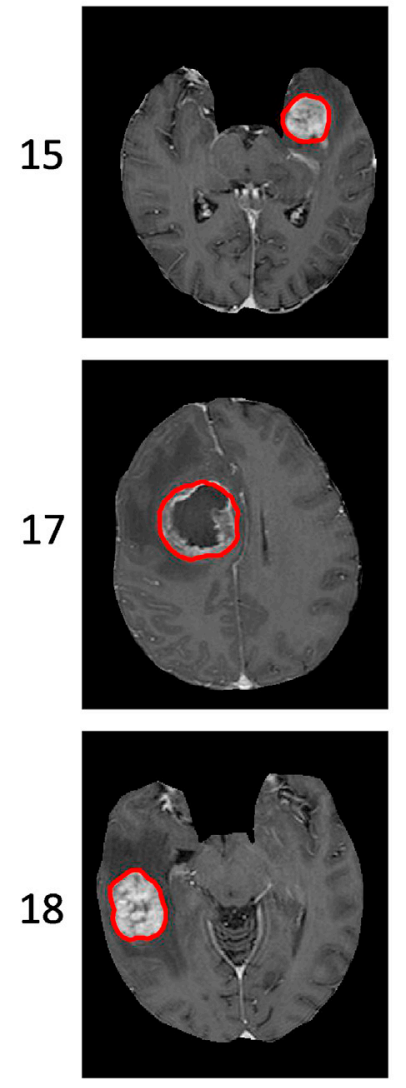

2: C-V
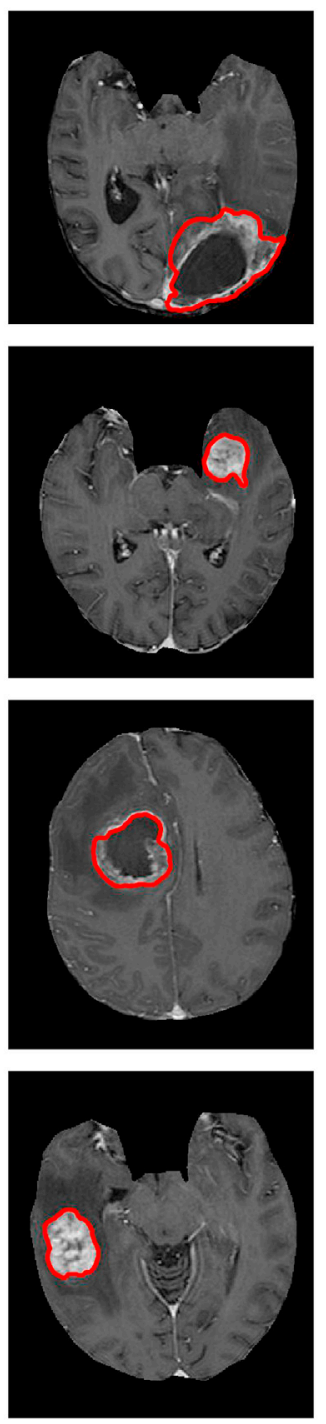

3: LACM-BIC
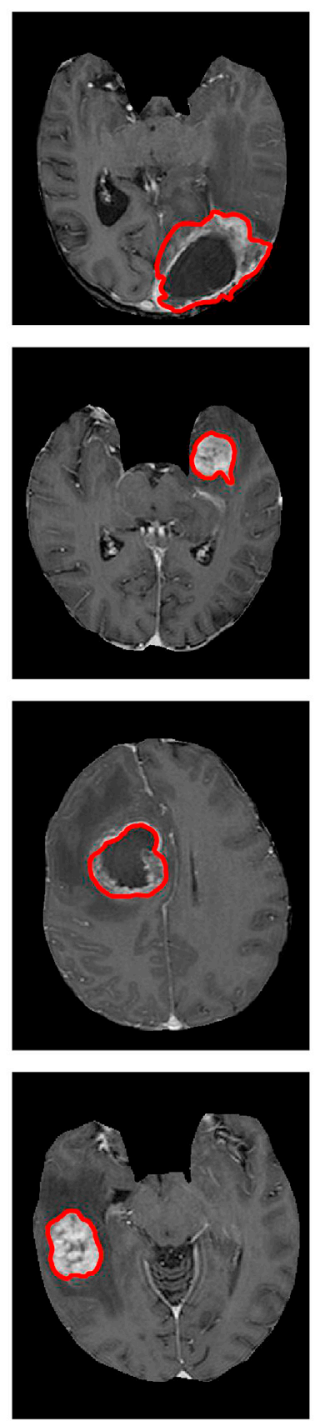

Selection
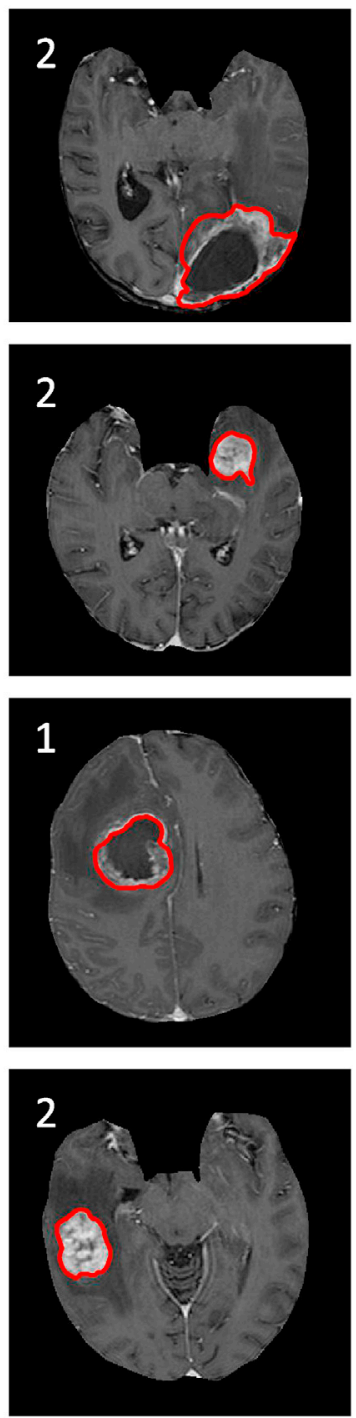

Fig. 7. Segmentation results of MRI data: patients 14, 15, 17 and 18. From left to right, the LGDF, localized C-V, LACM-BIC and the proposed algorithm.

\subsubsection{Feature extraction}

Statistical features are used to numerically describe images and they should provide information to represent an overall scene. If the computed features are chosen carefully to obtain the most representatives and discriminants, it is expected that the feature set will extract the relevant information from the image. These features allow performing the desired task exploiting a reduced representation instead of using the full image. The features considered for this work are mainly related to the description of statistical moments. Indeed, twelve features are selected and evaluated due to their capabilities to provide statistical intensity information necessary in the guidance of contour evolution. These twelve image features are listed in Table 1.

\subsection{Materials}

A total of 613 images were used in this study. These data are images from two datasets. Dataset one (or DB1) consists of a couple of MRI data from the MICCAI 2012 Challenge on Multimodal Brain Tumor Segmentation Benchmark (BRATS) database organized by B. Menze, A. Jakab, S. Bauer, M. Reyes, M. Prastawa, and K. Van Leemput (http://www.imm.
dtu.dk/projects/BRATS2012, [28]). Dataset two (or DB2) contains patient data collected at the Department of Neurosurgery at the University Hospital of Leipzig in Germany. Specifically, DB1 consists of 343 images i.e., 312 images from 11 patients with high-grade glioma taken into account in Ref. [20] and the additional 31 images carefully added to patients having the fewest slices (e.g., patients 2, 3, 6, 7 and 9). Dataset DB2 includes 270 images from 10 patients who have overcome a brain tumor surgery. Indeed, data from 21 patients are included in this work. They will be referred as patients 1 to 11 for DB1, and patients 12 to 21 for DB2. The global dataset (DB1 + DB2) was split into two parts, namely, training and testing data, respectively. The details of data as employed in the experiment are presented in Table 2.

\subsection{ACM initialization}

The ACM initialization is performed by using a binary shape descriptor based on hierarchical centroid. Proposed by Ref. [29] for handwriting recognition, this descriptor was used in Ref. [30] for automatic brain tumor detection in MRI data and it was referred as hierarchical centroid shape descriptor (HCSD). As in Refs. [31,32], for distinguishing structures based on their forms, the present approach considers that tumors have in general an ellipsoidal shape compared to 
other brain structures. The idea of this methodology is to compare the shape of binary structures extracted from input images to a prior shape considered as the reference. First, an HCSD is extracted from a cropped image containing a tumor with prior shape knowledge. Second, test images are binarized and then scanned by using a sliding window of $20 \%$ of the image size. The sliding window is moved forward to reach an overlap of $50 \%$ between adjacent windows in rows and columns. The HSCD is extracted from each window at every new position. Moreover, at the same time, the Euclidean distance is computed between the obtained descriptor and the reference. Finally, the tumor position is defined by the window that corresponds to the smallest distance. In a similar context, HCSD was applied to ACM initialization in Ref. [20]. However, in this last application, only the two biggest binary structures were retrieved from an image instead of scanning the entire image. Besides, their extracted HCSD allowed tumor structure detection. Therefore, the same technique is used in this work for LRACM initialization. The segmentation output of a slice provides the ACM initialization profile of the next one. But, after each four slices, a new tumor localization is then computed.

\section{Experimental results}

This section discusses the experimental results obtained in the segmentation of brain tumors with the proposed ACM selection system. Experiments were carefully conducted in two stages using the "BRATS2012" database (DB1) and the dataset from the University Hospital of Leipzig in Germany (DB2). In the first stage, different configurations of the suggested selection system were tested. Then, the application of the LRACM selector to brain tumors segmentation was assessed in the second stage. Two binary metrics were used to evaluate the segmentation results quantitatively, the Dice coefficient $(D)$ and the Hausdorff distance $\left(d_{H}\right)$. The perfect segmentation is reached when the measure $D \in[0,1]$ is equal to 1 and $d_{H}$ achieves a 0 value. These comparison metrics are computed as

$D=\frac{2|A \cap B|}{|A|+|B|}$

$d_{H}=\max \left\{\operatorname{maxmin}_{a \in A}\|a-b\|, \max _{b \in B} \min _{a \in A}\|b-a\|\right\}$

where $A$ is the handwork segmentation result (gold standard) and $B$ the automatically obtained contour.

\subsection{Selection system evaluation}

As already mentioned, the $K$-NN and RF methods were used for image classification by using the extracted features. Furthermore, the evaluation of the selection system was carried out by using the success rate (SR) [25] of image classification per patient. In contrast, the number of samples misclassified was assessed by using the failure rate (FR). Largely used in supervised learning, confusion matrices were used to compute the SR and FR for each patient. In the confusion matrix, the predictions are found in the columns and the ground truth (GT) class in the rows. GT indicates the patient data class based on the best ACM segmentation result reached. The percentages of classification rates are defined by

$S R=100 \times\left(N_{\text {correct }} / N\right)$

$F R=100 \times\left(N_{\text {wrong }} / N\right)$

where $N_{\text {correct }}, N_{\text {wrong }}$ and $N$ are the instances correctly classified, the instances misclassified and the total number of samples, respectively.

Three configurations were considered to analyze the behavior of the selection system (Fig. 3). First, the system was trained with a part of patients from DB1. The remaining of patients from DB1 and all patients from DB2 were used to test the system. Second, the system was trained with some of the patients from DB2 while the remain patients from DB2 and all patients from DB1 were used to test the system. Third, a part of patients from DB1 and DB2 are used to train the system whilst the rest of patients from DB1 and DB2 are used for testing. The first configuration showed that five and seven out of fourteen patients were successfully selected by using the RF and $k$-NN classifiers, respectively. In the second configuration, eight out of fifteen patients were properly classified by using both classifiers. Finally, in the last case, six out of eight patients were correctly selected. These results presented in Table 3 show that the first configuration allows to discriminate data from class 1 and class 3 whilst in the second one class 1 and 2 are the best discriminated. Therefore, the robustness of the selector is strongly dependent on the nature of the data used in the training stage. As a result, a high selection rate was achieved in the last case where data from DB1 and DB2 were mixed in the training step. Moreover, it can be observed that patients from all classes were represented in the result, unlike the other configurations. Based on these observations, the third configuration will be kept and employed in the selection of the best LRACM for brain tumor segmentation.

Figs. 4 and 5 present the confusion matrices of the patient image set classification by using the $k$-NN and RF classifiers, respectively. The bold values in the main diagonal represent all instances correctly classified $\left(N_{\text {correct }}\right)$. The row under each confusion matrix indicates the classification rate in percentage $\left(h_{i=z}^{m} / \sum_{i=1}^{3} h_{i}^{m}, z \in\{1,2,3\}\right)$ of image instances in each predicted class. The bold values represent the success rates (in percentage) achieved in the image classification process. The other values are the failure rates (in percentage and per class). The total FR is the sum of the failure rate of each class. Indeed, the selection made by the proposed system is successful when SR is the highest classification rate value for a specific patient. On the other hand, in the cases of system failure, the underlined value (FR) corresponds to the selected class.

Table 4 describes the successes and failures of the automatic selector for the patient classes compared to the ground truth by using the $k$-NN and RF classifiers. The class with the highest classification rate is selected as the expected prediction. Besides, the selection is successful when the prediction is equal to the GT. As already said, in this configuration six out of eight patients were properly chosen using the RF and $k$-NN methods. Nevertheless, the objectives of this study were not oriented to test classifiers but to the ACM selection system. Incidentally, another classifier (e.g., Adaboost, Bayesian-based classifiers, Neural Networks, etc.) can be integrated into this framework. Notice that their performances rely mainly on the training sample quality and the discriminant features extracted from the image [33-35].

Two classifiers were chosen to be tested in the suggested framework: the k-NN and RF algorithms. The k-NN is one of the main methods of classification and regression [36]. As a non-parametric algorithm, it does not make any assumptions about the data distribution. Hence, the k-NN classification method is commonly based on the Euclidean distance between a test sample and its $\mathrm{k}$ nearest neighbors from training samples. The RF is an ensemble based upon the basic premise that a set of classifiers do perform better classifications than an individual classifier does. In many classification tasks, the RF is known to be more accurate and robust to noise than single classifiers. Each classifier contributes with a single vote in the final class assignation of the test sample [37].

\subsection{Performance of the ACM selection system in brain tumor segmentation}

The aim of this work was to show that object segmentation by using ACM is image content dependent. A system for the selection of the best ACM based on image content was designed. Experiments were conducted in two main steps. Firstly, brain tumors were segmented in the image set of each patient by using the LGDF, C-V and LACM-BIC methods. Next, for the patients from the testing set, the selection of the best method among the three was performed. Table 5 presents the quantitative results 
reached by the ACM selection method and the ACM individually considered. These results come from the selection task achieved by the automatic selector and previously listed in Table 4. It is clear that, in the majority of cases, the best method for a specific patient is correctly selected by the system. Besides, we can notice that in both failure cases, the selection system chose the second best algorithm, which ensures a good final result. In order to have a global view of the outcomes, average values (mean \pm standard deviation) obtained by using the Local Binary Fitting (LBF) [38], LGDF [21], C-V [18], Local Mean Separation energy (LMS) [18], LACM-BIC [20] and the proposed selection system are showed in Table 6.

The results reveal that the proposed selection system achieves higher performance than comparative methods (i.e. $D=0.9320 \pm 0.0403$, $d_{H}=3.0025 \pm 1.4290$ ) with RF and $k$-NN classifiers. Figs. 6 and 7 depict the results achieved by using the LGDF, C-V, LACM-BIC and the selection system. The label of the selected active contour method is shown on the images in the selector column. Visual results sustain the quantitative evaluation where the LACM-BIC, followed by the C-V method, reached better performances than the LGDF model. Especially, in the case of patient 1 , it can be clearly observed that LACM-BIC is suitable for handling images where the target shared the border with the background and foreground.

\section{Discussion}

Accurate brain tumor segmentation is needed to quantitatively analyze the tumor and its growth over a period of time. Performed in preoperative MRI data, it enables to extract geometrical parameters like tumor volume, position, and distance to risk structures to plan the operation. In this study, an alternative framework for brain tumor segmentation in MR images is presented. This framework consists of a system of selection between three LRACM, namely, LGDF, C-V, and LACMBIC. Based on the content of input images, the system selects the LRACM that have to reach the best segmentation performance.

The experiments demonstrated that our system is able to select the ACM that should reach the optimal result. Hence, the average outcome from selected active contour models achieves better segmentation accuracy than the LBF [38], LGDF [21], C-V [18], LMS [18] and LACM-BIC [20] in brain tumor segmentation tasks. Two comparison metrics, the Dice coefficient, and Hausdorff distance were used to quantitatively sustain this claim. Thus, the results were compared to gold standards provided in the "BRATS-2012" database and by a medical expert from the Department of Neurosurgery at the University Hospital of Leipzig in Germany.

Since the system is based on supervised learning, its performance will depend on the outcomes reached in the classification step. In fact, a better image classification will improve the performance of the system. Reliable and commonly used, twelve image features and two classifiers $(k$-NN and $\mathrm{RF}$ ) were chosen. Not only have they been useful but they also allowed to show the effectiveness of the proposed system. It is important to note that the goal of this study was not to focus on testing classifiers but on the ACM selection system. Thus, other classifiers (e.g., Adaboost, Bayesianbased classifiers, Neural Networks, etc.) can be integrated into this framework. In the first experiments, three configurations of data splitting (training and testing sets) were used to analyze the behavior of the system. The outcomes demonstrated that the performance of the system changes for each configuration and showed that it depends mainly on the training sample quality and the discriminant features extracted from the image [33-35]. The LRAM selection applied to brain tumor segmentation was carried out by using the last configuration where the training set is composed of patient data from both databases (DB1 and DB2). A training set of heterogeneous data from different kinds of databases and tumors is important to make the system more robust. The second experiments were performed on the application of the LRACM selection system to the brain tumors segmentation. The selection using the RF and $k$-NN methods succeeded for six out of eight patients. The wrong selection of a LRACM can mean that the image features of the patient are not discriminative enough for these LRACM. Hence, the use of an adequate number of patients to train the system is important to overcome this problem. In the same fashion, the selection of the expected algorithm for the segmentation of a new input image should be improved. However, it can be noted that in the two cases of failure, the selection system chose the second best algorithm. In this way, it guarantees a good final result.

An important review of methods and results of the Multimodal Brain Tumor Image Segmentation Benchmark organized jointly with the MICCAI 2012 and 2013 conferences was presented in Ref. [39]. Several algorithms, considered as the state-of-the-art in brain tumor segmentation tasks, were evaluated in the discrimination of four types of intra-tumoral structures, namely edema, non-enhancing (solid) core, necrotic (or fluid-filled) core, and non-enhancing core. In this study, it was observed that fusing segmentations from different algorithms always performed better than the best individual algorithm applied to the same task. We recall that this observation is similar to the algorithm selection concept used in our work. It is important to note that, in contrast to [39], our work used a couple of data from BRATS 2012 dataset but it is not directly oriented to the MICCAI challenge. The aim of the suggested method is to focus the use of advantages of different ACM models in the segmentation task by selecting the optimal algorithm. So, the tumor segmentation is the chosen application to show how the selection of the best ACM algorithm for specific images achieves good segmentation results. Another difference is that our study considers only the tumor ? core? region (including all tumor structures except edema). Besides, the experiments in Ref. [40] revealed that Extremely randomized Trees classifier (similar to Random Decision Forests but introducing more randomness during the training phase) is among the most accurate in the classification of tumorous brain tissue. This statement sustains that supervised learning techniques achieve in general better results than un-supervised methods for tumor segmentation.

Our framework is easy to implement and it offers the benefit of being flexible. Applied on MRI data, the system showed its capability of selecting expected LRACM. Hence, it outperforms comparative methods in the segmentation of brain tumors. A suitable choice of features and classifiers is important to ensure a high accuracy rate in image segmentation. Images selected have in general tumors with an ellipsoidal shape as assumed in this study. The ACM initialization process used is limited to this kind of tumor shape. Based on the concept of the proposed system of automatic selection of LRACM, it can be applied to other medical imaging data, namely, Computed Tomography and ultrasound images. This framework can also be used in other applications of body organ segmentation including ventricles in cardiac images, prostate, lungs, and kidneys. Future efforts will be oriented toward the assessment of the proposed system in the use of 3D volumes, in the segmentation of images from other kinds of databases and using other active contour models.

\section{Conclusions}

The automatic selection of Localized region-based active contour model based on the image content and applied on brain tumor segmentation was presented in this work taking into account three LRACM for selection: the LGDF, C-V, and LACM-BIC. A system that selects the best LRACM among the three for segmenting brain tumor images from a specific patient is proposed. For the selection process, supervised learning techniques to classify images for each patient were used. Two well-known techniques, namely, the $k$-NN and Random Forest (RF) were used for the classification task. This framework offers the advantage of being flexible and modular, i.e., any classifier can be easily integrated, tested, and evaluated.

Results showed that the system properly selected six out of eight patients tested. Then, the suggested system of automatic selection of LRACM has been applied in the segmentation of brain tumor in MRI data. Indeed, several ACM were introduced in recent years and each of them claims to be effective under certain circumstances or particular cases. 
Faced with this reality and based on our observations and outcomes, in this study, experiments revealed that the performance of ACM is image content dependent. However, it is important to specify that our tests were performed only with localized active contour models and on MR brain tumor images. Furthermore, the outcomes revealed that the proposed selection system outperforms the performance of the three LRACM separately considered and two additional LRACM (LBF and LMS) included for comparison purposes. The most representative binary evaluation metrics were considered; the average accuracy values of $D=$ 0.9320 and $d_{H}=3.0025$ were reached by using the RF and $k$-NN classifiers, respectively. This framework can also be used in other applications of body organ segmentation including ventricles in cardiac images, prostate, lungs, and kidneys. Future work is needed to evaluate the proposed selection system by using different classes of ACM and other kinds of image databases.

\section{Acknowledgment}

This study has been supported by the Mexican National Council of Science and Technology (CONACyT) (grant number 493442). Besides, authors thank the University of Guanajuato for the financial support to conclude this study and especially to Professors Lankton and Wang that shared their source codes. Last, but not least, the authors acknowledge Dr. Dirk Lindner and, the University Hospital, Department of Neurosurgery, University of Leipzig for collecting the data in the context of a previous research project funded by the German Research Society (Deutsche Forschungsgemein-schaft) (grant number BU2336/1-1) and accepted by the Ethics Commission of the University of Leipzig.

\section{References}

[1] S. Pereira, A. Pinto, V. Alves, C.A. Silva, Brain tumor segmentation using convolutional neural networks in mri images, IEEE Trans. Med. Imaging 35 (5) (2016) 1240-1251, https://doi.org/10.1109/TMI.2016.2538465.

[2] M. Havaei, A. Davy, D. Warde-Farley, A. Biard, A. Courville, Y. Bengio, C. Pal, P.M. Jodoin, H. Larochelle, Brain tumor segmentation with deep neural networks, Med. Image Anal. 35 (2017) 18-31. https://doi.org/10.1016/j.media.2016.05.004. http://www.sciencedirect.com/science/article/pii/S1361841516300330.

[3] B.H. Menze, K.V. Leemput, D. Lashkari, T. Riklin-Raviv, E. Geremia, E. Alberts, P. Gruber, S. Wegener, M.A. Weber, G. Szkely, N. Ayache, P. Golland, A generative probabilistic model and discriminative extensions for brain lesion segmentation; with application to tumor and stroke, IEEE Trans. Med. Imaging 35 (4) (2016) 933-946, https://doi.org/10.1109/TMI.2015.2502596.

[4] M. Huang, W. Yang, Y. Wu, J. Jiang, W. Chen, Q. Feng, Brain tumor segmentation based on local independent projection-based classification, IEEE Trans. Biomed. Eng. 61 (10) (2014) 2633-2645, https://doi.org/10.1109/TBME.2014.2325410.

[5] A. Islam, S.M.S. Reza, K.M. Iftekharuddin, Multifractal texture estimation for detection and segmentation of brain tumors, IEEE Trans. Biomed. Eng. 60 (11) (2013) 3204-3215, https://doi.org/10.1109/TBME.2013.2271383.

[6] N. Cordier, H. Delingette, N. Ayache, A patch-based approach for the segmentation of pathologies: application to glioma labelling, IEEE Trans. Med. Imaging 35 (4) (2016) 1066-1076, https://doi.org/10.1109/TMI.2015.2508150.

[7] V.G. Kanas, E.I. Zacharaki, C. Davatzikos, K.N. Sgarbas, V. Megalooikonomou, A low cost approach for brain tumor segmentation based on intensity modeling and 3d random walker, Biomed. Signal Process. Control 22 (2015) 19-30. https://doi. org/10.1016/j.bspc.2015.06.004. http://www.sciencedirect.com/science/article/ pii/S1746809415001068.

[8] G. Vishnuvarthanan, M.P. Rajasekaran, P. Subbaraj, A. Vishnuvarthanan, An unsupervised learning method with a clustering approach for tumor identification and tissue segmentation in magnetic resonance brain images, Appl. Soft Comput. 38 (2016) 190-212. https://doi.org/10.1016/j.asoc.2015.09.016. http://www. sciencedirect.com/science/article/pii/S1568494615005888.

[9] E. Ilunga-Mbuyamba, J.M. Cruz-Duarte, J.G. Avina-Cervantes, C.R. Correa-Cely, D. Lindner, C. Chalopin, Active contours driven by cuckoo search strategy for brain tumour images segmentation, Expert Syst. Appl. 56 (2016) 59-68. https://doi.org/ 10.1016/j.eswa.2016.02.048. http://www.sciencedirect.com/science/article/pii/ S0957417416300872.

[10] E.A. Abdel Maksoud, M. Elmogy, 3D Brain Tumor Segmentation Based on Hybrid Clustering Techniques Using Multi-views of MRI, Springer International Publishing, Cham, 2016, pp. 81-104, https://doi.org/10.1007/978-3-319-33793-7_4. https:// doi.org/10.1007/978-3-319-33793-7_4.

[11] A. Hamamci, N. Kucuk, K. Karaman, K. Engin, G. Unal, Tumor-cut: segmentation of brain tumors on contrast enhanced $\mathrm{mr}$ images for radiosurgery applications, IEEE Trans. Med. Imaging 31 (3) (2012) 790-804, https://doi.org/10.1109/ TMI.2011.2181857.
[12] S. Bauer, R. Wiest, L.-P. Nolte, M. Reyes, A survey of mri-based medical image analysis for brain tumor studies, Phys. Med. Biol. 58 (13) (2013) R97. http://stacks. iop.org/0031-9155/58/i=13/a=R97.

[13] N. Gordillo, E. Montseny, P. Sobrevilla, State of the art survey on mri brain tumor segmentation, Magn. Reson. Imaging 31 (8) (2013) 1426-1438. https://doi.org/10. 1016/j.mri.2013.05.002. http://www.sciencedirect.com/science/article/pii/ S0730725X13001872.

[14] K. Saini, M.L. Dewal, M. Rohit, A fast region-based active contour model for boundary detection of echocardiographic images, J. Digital Imaging 25 (2) (2012) 271-278, https://doi.org/10.1007/s10278-011-9408-8. https://doi.org/10.1007/ s10278-011-9408-8.

[15] D. Gupta, R. Anand, B. Tyagi, A hybrid segmentation method based on gaussian kernel fuzzy clustering and region based active contour model for ultrasound medical images, Biomed. Signal Process. Control 16 (2015) 98-112. https://doi. org/10.1016/j.bspc.2014.09.013. http://www.sciencedirect.com/science/article/ pii/S1746809414001487.

[16] A. Pratondo, C.-K. Chui, S.-H. Ong, Integrating machine learning with region-based active contour models in medical image segmentation, J. Vis. Commun. Image Represent. 43 (2017) 1-9. https://doi.org/10.1016/j.jvcir.2016.11.019. http:// www.sciencedirect.com/science/article/pii/S1047320316302486.

[17] T. Chan, L. Vese, Active contours without edges, Image Process. IEEE Trans. 10 (2) (2001) 266-277, https://doi.org/10.1109/83.902291.

[18] S. Lankton, A. Tannenbaum, Localizing region-based active contours, Image Process. IEEE Trans. 17 (11) (2008) 2029-2039, https://doi.org/10.1109/ TIP.2008.2004611.

[19] A. Y Jr., A. Tsai, A. Willsky, A fully global approach to image segmentation via coupled curve evolution equations, J. Vis. Commun. Image Represent. 13 (12) (2002) 195-216. https://doi.org/10.1006/jvci.2001.0500.

[20] E. Ilunga-Mbuyamba, J.G. Avina Cervantes, A. GarciaPerez, R. de Jesus RomeroTroncoso, H. AguirreRamos, I. CruzAceves, C. Chalopin, Localized active contour model with background intensity compensation applied on automatic $\{\mathrm{MR}\}$ brain tumor segmentation, Neurocomputing 220 (2017) 84-97 recent Research in Medical Technology Based on Multimedia and Pattern Recognition, https://doi.org/10.1016/j.neucom.2016.07.057, http://www.sciencedirect.com/ science/article/pii/S092523121630902X.

[21] L. Wang, L. He, A. Mishra, C. Li, Active contours driven by local gaussian distribution fitting energy, Signal Process. 89 (12) (2009) 2435-2447 special Section: Visual Information Analysis for Security, https://doi.org/10.1016/j.sigpro. 2009.03.014.

[22] S. Takemoto, H. Yokota, Algorithm selection for intracellular image segmentation based on region similarity, in: 2009 Ninth International Conference on Intelligent Systems Design and Applications, (2009), pp. 1413-1418. doi:10.1109/ ISDA.2009.205

[23] M. Lukac, K. Abdiyeva, M. Kameyama, Evaluation of algorithm selection approach for semantic segmentation based on high-level information feedback, in: 2015 International Conference on Information and Digital Technologies, 2015, pp. 223-229. doi:10.1109/DT.2015.7222974.

[24] M. Lukac, K. Abdiyeva, M. Kameyama, Symbolic segmentation using algorithm selection, CoRR abs/1505.07934. URL http://arxiv.org/abs/1505.07934.

[25] J. Cepeda-Negrete, R.E. Sanchez-Yanez, Automatic selection of color constancy algorithms for dark image enhancement by fuzzy rule-based reasoning, Appl. Soft Comput. 28 (2015) 1-10. https://doi.org/10.1016/j.asoc.2014.11.034. http:// www.sciencedirect.com/science/article/pii/S1568494614005961.

[26] P.W. Tse, D. Wang, The automatic selection of an optimal wavelet filter and its enhancement by the new sparsogram for bearing fault detection: Part 2 of the two related manuscripts that have a joint title as two automatic vibration-based fault diagnostic methods using the novel sparsity measurementparts 1 and 2, Mech. Syst. Signal Process. 40 (2) (2013) 520-544. https://doi.org/10.1016/j.ymssp.2013.05. 018. http://www.sciencedirect.com/science/article/pii/S088832701300277X.

[27] G. Cano, J. Garcia-Rodriguez, A. Garcia-Garcia, H. Perez-Sanchez, J.A. Benediktsson, A. Thapa, A. Barr, Automatic selection of molecular descriptors using random forest: application to drug discovery, Expert Syst. Appl. 72 (2017) 151-159. https://doi.org/10.1016/j.eswa.2016.12.008. http://www.sciencedirect. com/science/article/pii/S0957417416306819.

[28] B. Menze, A. Jakab, M. Reyes, S. Bauer, M. Prastawa, K.V. Leemput (Eds.), Proceedings of the MICCAI Challenge on Multimodal Brain Tumor Image Segmentation (BRATS) 2012, MICCAI, Nice, France, 2012. https://hal.inria.fr/hal00912935.

[29] S. Armon, Handwriting Recognition and Fast Retrieval for Hebrew Historical Manuscripts (Master's thesis), The Hebrew University of Jerusalem, 2011.

[30] E. Ilunga-Mbuyamba, J. G. Avina-Cervantes, D. Lindner, J. Guerrero-Turrubiates, C. Chalopin, Automatic brain tumor tissue detection based on hierarchical centroid shape descriptor in tl-weighted mr images, in: 2016 International Conference on Electronics, Communications and Computers (CONIELECOMP), 2016, pp. 62-67. doi:10.1109/CONIELECOMP.2016.7438553.

[31] P.R. Jethwa, J.H. Lee, R. Assina, I.A. Keller, S.F. Danish, Treatment of a supratentorial primitive neuroectodermal tumor using magnetic resonanceguided laser-induced thermal therapy, J. Neurosurg. Pediatr. 8 (5) (2011) 468-475 pMID: 22044371, https://doi.org/10.3171/2011.8.PEDS11148.

[32] J.J. Corso, E. Sharon, S. Dube, S. El-Saden, U. Sinha, A. Yuille, Efficient multilevel brain tumor segmentation with integrated bayesian model classification, IEEE Trans. Med. Imaging 27 (5) (2008) 629-640, https://doi.org/10.1109/ TMI.2007.912817.

[33] P. Du, A. Samat, B. Waske, S. Liu, Z. Li, Random forest and rotation forest for fully polarized $\{S A R\}$ image classification using polarimetric and spatial features, \{ISPRS\} Journal of Photogrammetry and Remote Sensing 105 (2015) 38-53. 
http://doi.org/10.1016/j.isprsjprs.2015.03.002. http://www.sciencedirect.com/ science/article/pii/S0924271615000611.

[34] P. Olofsson, G.M. Foody, M. Herold, S.V. Stehman, C.E. Woodcock, M.A. Wulder, Good practices for estimating area and assessing accuracy of land change, Remote Sensing of Environment 148 (2014) 42-57. http://doi.org/10.1016/j.rse.2014.02. 015. http://www.sciencedirect.com/science/article/pii/S0034425714000704.

[35] Z. Sun, G. Bebis, R. Miller, Monocular precrash vehicle detection: features and classifiers, IEEE Transactions on Image Processing 15 (7) (2006) 2019-2034, https://doi.org/10.1109/TIP.2006.877062.

[36] J. Fritz, Distribution-free exponential error bound for nearest neighbor pattern classification, IEEE Transactions on Information Theory 21 (5) (1975) 552-557, https://doi.org/10.1109/TIT.1975.1055443.

[37] V. Rodriguez-Galiano, B. Ghimire, J. Rogan, M. Chica-Olmo, J. Rigol-Sanchez, An assessment of the effectiveness of a random forest classifier for land-cover classification, ISPRS Journal of Photogrammetry and Remote Sensing 67 (2012) 93-104. https://doi.org/10.1016/j.isprsjprs.2011.11.002. http://www. sciencedirect.com/science/article/pii/S0924271611001304.

[38] C. Li, C. Y. Kao, J. C. Gore, Z. Ding, Implicit active contours driven by local binary fitting energy, in: 2007 IEEE Conference on Computer Vision and Pattern Recognition, 2007, pp. 1-7. doi:10.1109/CVPR.2007.383014.

[39] B.H. Menze, A. Jakab, S. Bauer, J. Kalpathy-Cramer, K. Farahani, J. Kirby, Y. Burren, N. Porz, J. Slotboom, R. Wiest, L. Lanczi, E. Gerstner, M.A. Weber, T. Arbel, B.B. Avants, N. Ayache, P. Buendia, D.L. Collins, N. Cordier, J.J. Corso, A. Criminisi, T. Das, H. Delingette, Demiralp, C.R. Durst, M. Dojat, S. Doyle, J. Festa, F. Forbes, E. Geremia, B. Glocker, P. Golland, X. Guo, A. Hamamci,

K.M. Iftekharuddin, R. Jena, N.M. John, E. Konukoglu, D. Lashkari, J.A. Mariz, R. Meier, S. Pereira, D. Precup, S.J. Price, T.R. Raviv, S.M.S. Reza, M. Ryan, D. Sarikaya, L. Schwartz, H.C. Shin, J. Shotton, C.A. Silva, N. Sousa, N.K. Subbanna, G. Szekely, T.J. Taylor, O.M. Thomas, N.J. Tustison, G. Unal, F. Vasseur,

M. Wintermark, D.H. Ye, L. Zhao, B. Zhao, D. Zikic, M. Prastawa, M. Reyes, K.V. Leemput, The multimodal brain tumor image segmentation benchmark (brats), IEEE Transactions on Medical Imaging 34 (10) (2015) 1993-2024, https://doi.org/ 10.1109/TMI.2014.2377694.

[40] M. Goetz, C. Weber, J. Bloecher, B. Stieltjes, H.-P. Meinzer, K. Maier-Hein, Extremely randomized trees based brain tumor segmentation, in: In Proc of BRATS Challenge - MICCAI, 2014.

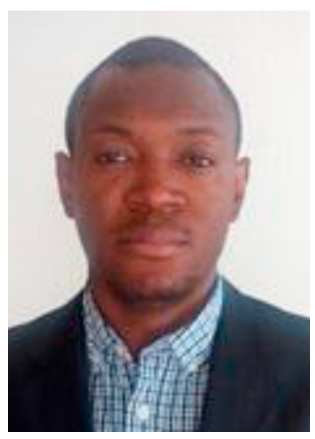

Elisee Ilunga Mbuyamba received the B.Eng. degree in Electrical Engineering from the Institut Supérieur de Techniques Appliquées (ISTA, Kinshasa, DR Congo) in 2006. Then, he obtainedthe M.Eng. and the Ph.D. degrees in Electrical Engineering from the Universidad de Guanajuato in 2014 and 2017, respectively. His research interests include machine learning, pattern recognition and medical image processing.

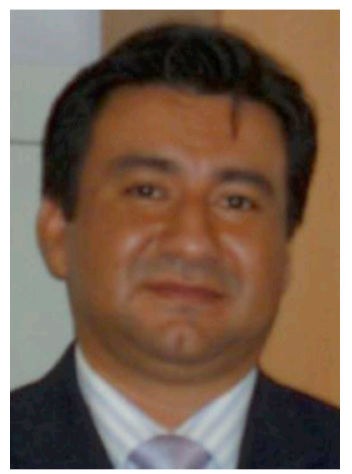

Juan Gabriel Avina-Cervantes received the B.Eng. degree in Electronics and Communications Engineering from the Universidad de Guanajuato in 1998, the M. Eng. degree in Electrical Engineering (Instrumentation and Digital Systems) from the same University in 1999, the Ph.D. degree in Informatics and Telecommunications from the Institut
National Polytechnique de Toulouse and the LAASCNRS, France, in 2005. His research interests include artificial vision for outdoor mobile robotics, pattern recognition, optimal control systems and image processing. Currently, he is a researcher and full-time professor at the Universidad de Guanajuato.

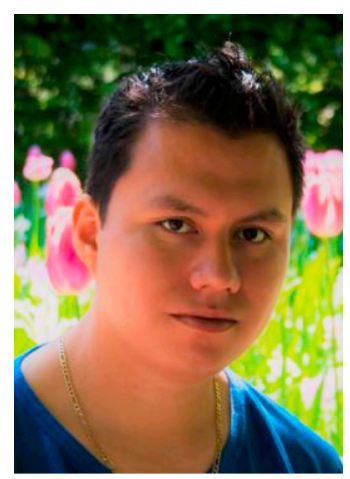

Jonathan Cepeda-Negrete is a Full-time professor at the Department of Agricultural Engineering from the Life Sciences Division, University of Guanajuato. $\mathrm{He}$ received his $\mathrm{PhD}$ in Electrical Engineering (2016), his Master in Electrical Engineering (2012) and his Bachelor in Engineering of Communications and Electronics (2011) from the University of Guanajuato, Mexico. His current research interests include computer vision applications, mainly color image processing, pattern recognition, intelligent systems and automatization of agricultural processes.

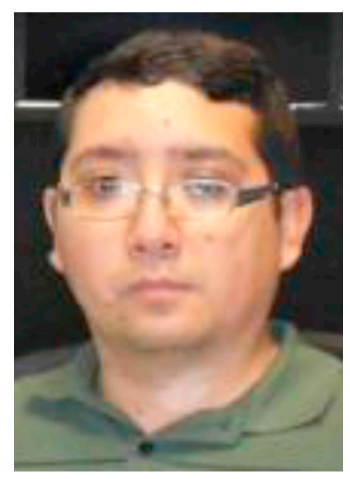

Mario Alberto Ibarra Manzano obtained his B.Eng. degree in communication and electronic engineering in 2003 and his M.Eng. degree in Electric in 2006 from the University of Guanajuato, Salamanca, Mexico. In 2011, he obtained his PhD degree (with honors) from the Institut National des Sciences Appliquées, Toulouse, France. He is interested in digital design on FPGA for image processing applied on autonomous robots and real-time systems. Currently, he is assistant professor at Universidad de Guanajuato in the Electronics Engineering Department.

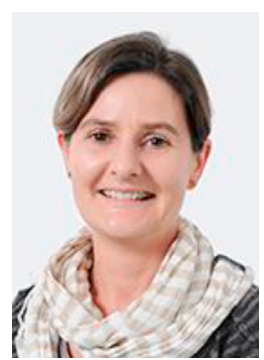

applications.
Claire Chalopin, received the Ph.D. degree in 2000 in Medical Imaging from the University of Lyon, France. She worked as Research Follow on the segmentation of focal brain lesions at the Max Planck Institute for Human Cognitive and Brain Sciences in Leipzig, Germany. She reached in 2006 ICCAS at the University of Leipzig, and developed new methods to extract vascular structures and brain tumors in ultrasound image data acquired in the operating room during brain surgeries. Since 2015 she is developing her own research group "Multimodal Intraoperative Imaging". Her research area focuses on the evaluation and improvement of innovative non-invasive imaging modalities, like ultrasound imaging and infrared thermography, for surgical 